\title{
LTE and IEEE 802.11p for vehicular networking: a performance evaluation
}

\author{
Zeeshan Hameed Mir ${ }^{*}$ and Fethi Filali
}

\begin{abstract}
Various wireless communication systems exist, which enable a wide range of applications and use cases in the vehicular environment. These applications can be grouped into three types, namely, road safety, traffic efficiency, and infotainment, each with its own set of functional and performance requirements. In pursuance of assisting drivers to travel safely and comfortably, several of these requirements have to be met simultaneously. While the coexistence of multiple radio access technologies brings immense opportunities towards meeting most of the vehicular networking application requirements, it is equally important and challenging to identify the strength and weaknesses of each technology and understand which technology is more suitable for the given networking scenario. In this paper, we evaluate two of the most viable communication standards, Institute of Electrical and Electronics Engineers (IEEE) 802.11p and long-term evolution (LTE) by 3rd Generation Partnership Project for vehicular networking. A detailed performance evaluation study of the standards is given for a variety of parameter settings such as beacon transmission frequency, vehicle density, and vehicle average speed. Both standards are compared in terms of delay, reliability, scalability, and mobility support in the context of various application requirements. Furthermore, through extensive simulation-based study, we validated the effectiveness of both standards to handle different application requirements and share insight for further research directions. The results indicate that IEEE $802.11 \mathrm{p}$ offers acceptable performance for sparse network topologies with limited mobility support. On the other hand, LTE meets most of the application requirements in terms of reliability, scalability, and mobility support; however, it is challenging to obtain stringent delay requirements in the presence of higher cellular network traffic load.
\end{abstract}

Keywords: IEEE 802.11 p; LTE; ITS; Vehicular applications; VANET

\section{Introduction}

Many believe that the integration of information and communication technologies with transportation infrastructure and vehicle will revolutionize the way we travel today. The enabling technologies envisioned to realize the proposed framework would spur an array of applications and use cases in the domain of road safety, traffic efficiency, and infotainment. These applications allow dissemination and gathering of useful information among vehicles and between transportation infrastructure and vehicles in pursuance of assisting drivers to travel safely and comfortably. Reliable and low-latency communication between vehicles and transport infrastructure is critical to the implementation success of many of these applications.

\footnotetext{
* Correspondence: zeeshanh@qmic.com

Qatar Mobility Innovations Center (QMIC), Qatar Science and Technology Park (QSTP), PO Box 210531, Doha, Qatar
}

Vehicular networking is the enabling technology which allows the realization of the variety of applications and use cases. Given the significant potential to cater for diverse applications and their performance requirements, there has been a growing demand to equip vehicles with multiple connectivity modalities. In order to fully exploit these capabilities, vehicles are required to intelligently select the most appropriate technology for the specific networking scenarios. To this end, it is necessary to know the strengths and weaknesses of each technology and understand which technology would be more suitable. Among several communication modalities, in this paper, we choose to compare Institute of Electrical and Electronics Engineers (IEEE) 802.11p and long-term evolution (LTE) for various networking conditions, operating parameter settings, and application requirements.

The IEEE 802.11p [1] is considered as the de facto standard to implement several of the vehicular networking 
applications. The standard includes Physical (PHY) and Medium Access Control (MAC) layer specification as well as upper-layer protocols. IEEE 802.11 p is essentially an IEEE 802.11-based standard adapted for the wireless environment with vehicles. It inherits several of the characteristics like simplicity and distributed medium access control mechanism. Mostly, in-vehicle on-board units (OBUs) and roadside units (RSUs) fixed with transport infrastructure like traffic signals utilize this standard. Given the diverse performance requirements from a wide spectrum of vehicular networking applications, several advocate the feasibility of LTE standard by the 3rd Generation Partnership Project (3GPP) [2] as an emerging solution. To this end, we envision an advanced LTE-technology-enabled OBU. Alternatively, the smartphones with LTE connectivity can be leveraged to support a number of applications [3].

The main objective of this paper is to conduct a comparative study between IEEE 802.11p and LTE and evaluate their suitability to different vehicular networking applications. The novelty of our study lies in the exploration of the impact of variety of networking parameters settings such as beacon transmission frequency, vehicle densities, and average vehicle speed on the performance. Both standards are compared in terms of delay, reliability, scalability, and mobility support while accounting for different vehicular networking application requirements in the domain of road safety, travel efficiency, and infotainment. Additionally, we also include a summary of the IEEE 802.11p and LTE standards, an overview of the vehicular networking applications and their performance requirements, and a detailed discussion on the lessons learned and insight for future research directions. Moreover, the use of single simulation platform for modeling and evaluation makes this study useful for more realistic and applied systems.

In order to clearly state the overall scope of the paper, we define the following research questions which are addressed in this paper:

(1). How do different networking parameters such as beaconing frequency, vehicle density, and vehicle average speed affect the performance of IEEE 802.11p and LTE?

(2). For what settings of parameter values that the performance of IEEE 802.11p and LTE degrades against a set of vehicular networking application requirements?

(3). Does the performance in terms of delay, reliability, scalability, and mobility support degrade significantly or trivially with the change in different parameter values?

(4). What types of applications would be supported by IEEE 802.11p and LTE?
There are number of research studies which assess the feasibility of IEEE 802.11p and LTE standards to support vehicular networking applications. However, to the best of our knowledge, most of the previous studies only answer a subset of the above-mentioned questions or partially cover the topics presented in this paper.

The comparison between two fundamentally differently designed standards (i.e., infrastructure-less vs. infrastructure-based) under various networking conditions and performance requirements reveals interesting insight. Our results indicate that while the infrastructureless access domain covers the typical requirements of simplicity and latency, to some extent, the decentralized nature of IEEE 802.11p imposes limitations on the reliability of the standard mainly originated from the ad hoc or uncoordinated medium access mechanism, severe congestion due to higher beaconing frequency, and higher vehicle mobility. Another issue is of scalability, where the performance tends to degrade as the number of participants increases. On the other hand, while the improved performances, reliability, scalability, and mobility support are the hallmark of LTE technology, it is challenging to obtain stringent latency requirements over the cellular connection especially in the presence of higher cellular network traffic load.

The rest of this paper is organized as follows. Section 2 describes the related work and explains the state-of-theart and its limitations. We provide an overview of the IEEE 802.11p standard and LTE technology and their application in the vehicular networking domain in Section 3. In Section 4, we present the taxonomy of vehicular network applications along with their performance requirements. The performance evaluation is described in Section 5. In Section 6 we position our work among previous similar studies and establish its relevance and limitations. Finally, we conclude the paper in Section 7.

\section{Related work}

This section summarizes the studies which evaluated the technical feasibility of IEEE 802.11p and 3G/4G cellular networks to support the vehicular networking applications.

Studies on IEEE 802.11p can be largely divided in to three categories, i.e., analytical modeling, simulation, and testbed-oriented [4]. Those falling into the first category include [5-8]. Broadly, the authors investigated throughput, collision probability, maximum range, and delay with number of traffic and networking conditions. In these studies, the Markov chain analytical models are often followed by simulation-based validations. Work based on simulation studies are described in [9-12]. The authors presented detailed simulation results covering several general features of the IEEE 802.11p and discussed 
various performance and simulation parameters under variety of simulation scenarios. More notably, the work in [12] includes a comparison between IEEE 802.11p and WiMAX technology and measures the impact of varying data rate and vehicle speed on both standards. This paper extends on the previous preliminary efforts given in $[13,14]$ to check the feasibility of infrastructure-based WiMAX standard in the vehicular networking environment. Additionally, a numbers of papers have evaluated the IEEE $802.11 \mathrm{p}$ performance using the real implementation of the standard [15-17]. The authors demonstrated vehicular communication scenarios with both urban and rural settings.

More recently, several standardization bodies and research consortium have shown increasing interest in adopting LTE technology to support vehicular networking applications [18]. In this regard, the feasibility of the LTE technology has been comprehensively studied, and a number of framework and prototype implementations can also be found in the literature. For example, in [19] authors advocate the suitability of LTE (4G) over HSUPA (3G) cellular networks. Both standards were tested on real-time testbed for various vehicle speeds. It is shown that LTE is capable of satisfying delay requirements for most of the vehicular applications. Similarly, authors in [20] combined the LTE and cloud-based architecture into a unified framework to offset the scalability issues. By locating server closer to the vehicles and multicast/broadcast downlink communication techniques, the proposed framework improves the effectiveness of the road safety application. More closely related to this paper is Trichias' work [21] on the feasibility study of LTE technology for the intelligent transportation systems (ITS). There are mainly two parts of the work including performance evaluation of LTE for various scheduling schemes and comparison with the IEEE 802.11p standard. While the author tries to find answers to a set of very valid and interesting questions, the work only covers a high-level comparison, lacking a detailed study. Some other limiting factors include use of rudimentary simulation environment modeling with several of the critical technical features missing from the implementation of both standards. Moreover, two different simulation platforms were used for the study without taking into account for the implementation differences. On a more analytical perspective, Vinel $[22,23]$ evaluated both standards using a theoretical framework, while Phan et al. [24] provided the capacity analysis for LTEbased vehicular network. The presented results are meaningful because they provided the analytical perspective of the cellular-network-based vehicular network performance. However, the main focus of both studies was on the delay and the scalability issues with no or little consideration for the reliability and mobility support.
Moreover, these studies only cater the performance requirements of road safety applications.

Higher penetration level, ease in testing and deployment, multiple connectivity modalities, and several built-in sensors motivated Park et al. to develop VoCell [3], a smartphone-based vehicular networking application framework. The proposed framework utilizes smartphones to exchange vehicle and sensory information among a set of applications. The design of the VoCell architecture incorporates many of the required features observed through comprehensive empirical study of IEEE 802.11p/wireless access in vehicular environment (WAVE) and LTE-based vehicular networks. Similarly, authors in [25] proposed a software framework for designing and developing vehicular network applications on smartphones. The proposed architecture exploits several communication technologies such as Bluetooth, Wi-Fi Direct, and 3G/4G. In [26], two frameworks were proposed to develop, test, and deploy vehicular network applications. The smartphone-based and laptop-based frameworks provide easily available platforms to emulate an ad hoc network; however, the AP (access point) has to suffer higher network load and communication overhead. In [27], authors described a smartphone-based road safety application which disseminates warning messages. The uses of smartphones result in real-time testing and deployment at lower cost, while the location estimates error which remains within the prescribed limits. Abid et al. [28] present the smartphone adaptation for vehicular network applications. While the focus is on vehicle-to-transportation infrastructure communication over the LTE networks, no comparative study with IEEE $802.11 \mathrm{p}$ standard is provided. The simulation results cover latency, throughput, and packet loss ratio. Corti et al. [29] evaluated the performance of UMTS cellular network in a real vehicular network. Their work describes a centralized road safety application named advanced driver assistance system (ADAS) and only focus on the cellular network latency and centralized server response delay of the proposed architecture.

Furthermore, the notion of hybrid networks for the vehicular networking has also been evaluated to combine the best form infrastructure-less and infrastructure-based access domains. Zhao et al. [30] proposed 3G cellularnetwork-assisted data delivery for vehicular ad hoc networks (VANETs). The approach leverages on a utility function that controls the interplay between data delivery ratio, delay, and $3 \mathrm{G}$ traffic budget. The effectiveness of the proposed scheme is evaluated through empirical study based on real traces. In [31], Busanelli et al. present a heterogeneous network architecture for smartphone-based information dissemination. The communication is divided between two types of vehicles, where primary vehicles take on the role as a gateway of information from 3G/4G cellular network, while secondary vehicles 
exchange information via IEEE 802.11b-based ad hoc network. In [32] and [33], authors assumed in-vehicle OBUs with multiple interfaces and proposed LTE technology assisted cluster-head selection and management protocols for the IEEE 802.11p-based VANETs. Karsaros et al. [34] proposed heterogeneous architecture for location service which offloads the location service control traffic over the infrastructure-based LTE cellular networks while utilizing IEEE 802.11p ad hoc network for transferring data traffic. All these protocols have showed considerable performance improvements over the use of single access technology for the vehicular networking, which consequently further justifies the need of comprehensive study into the feasibility of one over the other.

\section{Vehicular networking with IEEE 802.11p and LTE}

Vehicular networking combines wireless communication, in-vehicle sensing module, and Global Positioning System (GPS) to enable a variety of applications in road safety, traffic efficiency, and infotainment domains. The current technologies are based on DSRC (dedicated short-range communications) global standard. In 1999, the FCC allocated $75 \mathrm{MHz}$ of spectrum at 5.850 to $5.925 \mathrm{GHz}$ frequency range. In the USA, the total bandwidth is divided in to seven $10-\mathrm{MHz}$ bandwidth channels mainly of two types, i.e., control channel $(\mathrm{CCH})$ and service channels (SCHs), while the remaining $5 \mathrm{MHz}$ is dedicated as the guard band. The control channel $(\mathrm{CCH})$ is dedicated for broadcasting short messages and communication management for the road safety applications, whereas the service channels (SSHs) is reserved for the traffic efficiency and infotainment applications.

In order to widely spread these technologies, standardization at each layer of the networking protocol stacks has to be done. Therefore, a suite of protocols along with the architecture for the wireless environments with vehicles is developed called wireless access in vehicular environment or WAVE.

The WAVE protocol stack is composed of several components, most notably the IEEE $802.11 \mathrm{p}$ which is essentially an IEEE 802.11-based standard adapted for the vehicular networking environment. The noteworthy characteristic of the $\mathrm{PHY}$ layer is the reduction to $10 \mathrm{MHz}$ bandwidth from IEEE 80.11a $20 \mathrm{MHz}$, which halves the data rate to 3 to $27 \mathrm{Mbps}$ from 6 to $54 \mathrm{Mbps}$. The IEEE 802.11p MAC is 802.11e Enhanced Distributed Channel Access (EDCA) with quality of service (QoS) support. The PHY layer and the MAC layer together allow ad hoc communication among OBUs as well as between OBUs and the RSUs. The absence of infrastructureassisted channel access mechanism and wireless communication among high-speed vehicles over the varying channel condition possesses significant challenges in terms of overall network performances.

On the other side of the spectrum, the LTE standard by 3GPP offers superior performances in terms of throughput and lower latencies. Theoretically, downlink data rate of $150 \mathrm{Mbps}$ and uplink data rate of $50 \mathrm{Mbps}$ is achievable in a $20-\mathrm{MHz}$ downlink and uplink spectrum, respectively. This is combined with the latency of less than $5 \mathrm{~ms}$ in the user plane. The cost and performance efficiency aspect of the LTE technology are due to the simplification in network architecture (fewer network elements) and advanced algorithms for resource utilization. The base station or eNodeB (also eNB) comprises the radio access network (RAN) of the LTE network architecture which takes care of all radio control and management functionalities and the interaction between the user equipment (UE) and LTE core network. The eNB is directly connected with the Evolved Packet Core (EPC) which supports mobility management, QoS handling and interoperability with legacy 3GPP, and non3GPP access technologies.

The LTE standard specification not only promises users a truly broadband experience but also enables mobile network operators (MNOs) to deliver sophisticated services in an effective manner. It has been envisioned to exploit the very existing LTE infrastructure to support vehicular networking applications either through an advanced LTE-enabled OBU or using smartphones with LTE connectivity. However, the key challenge is to deliver time-constraint data over the 4G/LTE connection and efficiently share resources with the mobile users.

In this paper we propose to compare two architectures for vehicular networking which utilizes either IEEE $802.11 \mathrm{p}$-based infrastructure-less network or infrastructure-based LTE cellular network. An urban scenario for these architectures is considered as illustrated in Figure 1. The left-hand side depicts IEEE 802.11p-based vehicular network where multiple RSUs are connected to a gateway through a backbone network which in turn provides access to the Internet, whereas the right-hand side shows another approach for vehicular networking where the LTE-enabled OBUs or smartphones exploit the already existent LTE cellular infrastructure to communicate over the wireless medium. From the data flow perspective, in-vehicle OBU or smartphone samples and gathers the relevant information and periodically exchanges beacon messages with other vehicles either directly or via RSUs in an ad hoc manner. Alternatively, this exchange is carried out through the base station node (eNB in LTE) of the cellular network. In the latter case, all the beacons that are received at the eNB has to traverse the entire core network (EPC) before it can be disseminated to the rest of the vehicles in the network. It is assumed that the eNB sends the beacon messages via 


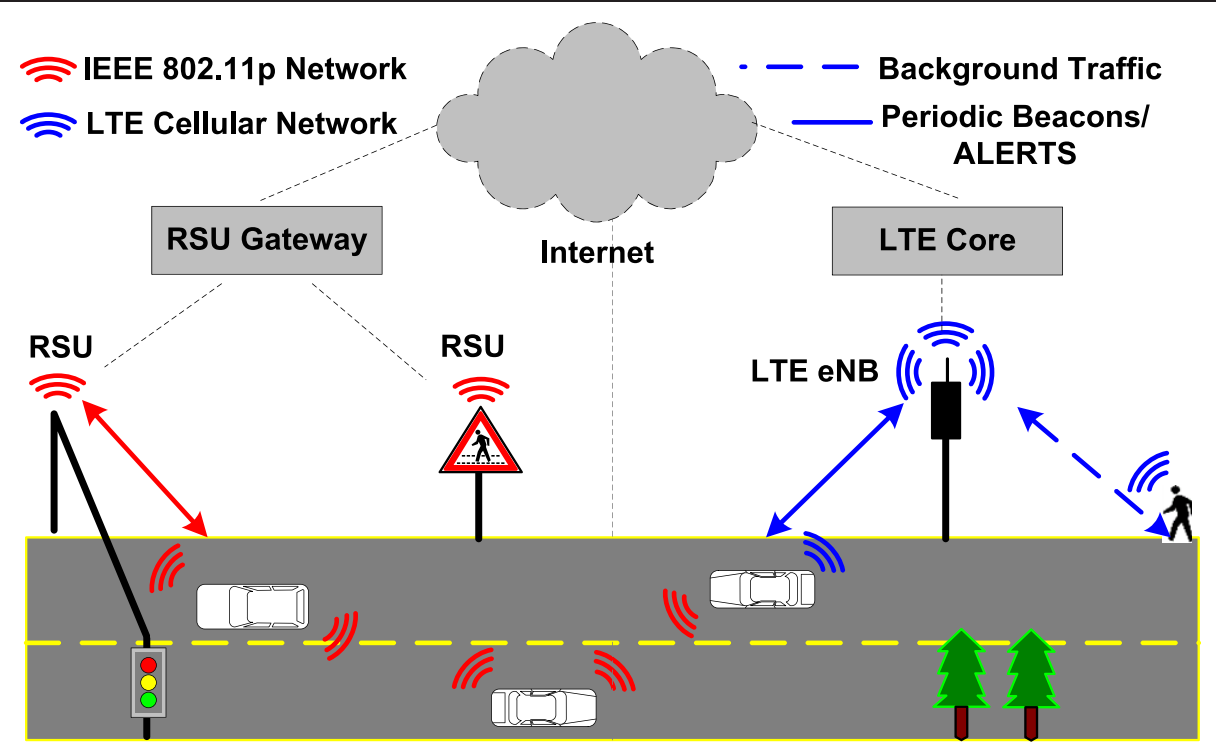

Figure 1 Vehicular networking scenario using IEEE $802.11 \mathrm{p}$ and LTE.

multiple unicast transmissions. Although other effective techniques such as Multimedia Broadcast Multicast Service (MBMS) can be utilized, its use is explored in our future work. In order to make the scenario realistic, the LTE network caters for both downlink and uplink mobile phone users (termed as background traffic) [21,34] along with the vehicular networking traffic.

\section{Vehicular networking applications and requirements}

The integration of information and communication technology with transport infrastructure and vehicles would enable an array of vehicular safety, traffic efficiency, and infotainment applications. These applications would help in significantly reducing the number and severity of road accidents, improve traffic management, and enhance passenger convenience. While there are several types of functional and performance requirement, the main focus of this paper is on the system performance such as minimum permissible delay, range (i.e., coverage distance), data rate and data notification, or transmission frequency [35-37]. In order to provide efficient network functionality, several of these requirements have to be met simultaneously:

(1). Active road safety applications: Enabled by a set of detailed data both from local sensor measurements and obtained from other participants within the vehicular network, these applications provide drivers with an unprecedented, extensive field of view on the driving environment. The goal is to decrease the probability of injuries in general and, ultimately, to reduce the number of traffic deaths to a minimum. System performance requirements of active road safety applications include lower latency $(\leq 100 \mathrm{~ms})$, short to long coverage distance (300 $\mathrm{m}$ to $20 \mathrm{~km}$ ), minimum transmission frequency of $10 \mathrm{~Hz}$, and low-to-medium data rates (1 to $10 \mathrm{kbps}$ ).

(2). Cooperative traffic efficiency: Cooperative traffic efficiency applications are intended to provide additional information via wireless communication links to improve the traffic flow, to enhance the traffic coordination and management, and to reduce the environmental impact. Traffic regulation messages will typically be triggered by traffic management entities controlled by the authorities. System performance requirements of cooperative traffic efficiency applications include medium latency ( $\leq 200 \mathrm{~ms})$, short to medium coverage distance ( $300 \mathrm{~m}$ to $5 \mathrm{~km}$ ), minimum transmission frequency between 1 and $10 \mathrm{~Hz}$, and data rates from one to tens of kbps.

(3). Infotainment: Infotainment applications provide the user with information to enhance the passenger comfort/convenience or enable global Internet services. System performance requirements of infotainment applications include relatively longer delay $(\leq 500 \mathrm{~ms})$, short to long coverage distance which can varies from a few meters to full communication range, minimum transmission frequency of $1 \mathrm{~Hz}$, and data rates of one to tens or several hundreds of kbps for data transmission (depending on the contents).

Given numerous vehicular networking applications and use cases with diverse set of systems performance 
requirements, it is difficult for a single standard to accommodate all of them simultaneously, specially, when most of these requirements are often conflicting in nature. For instance, the active road safety applications require high level of real-time data acquisition and processing. This is necessary to allow drivers to react towards any hazardous events in an appropriate way within a critical time window. Using high-precision geo-spatial position information and lower latency wireless technology, drivers can be warned timely of any potential dangerous situation. In these scenarios, an obvious solution would be to employ IEEE 802.11p-based direct communication either through roadside infrastructure or between vehicles so that the potential accident can be averted. However, to generate reliable and delay-constrained warning notifications under certain environment can be challenging. For example, in an urban area with several high-rise buildings or intersections, line of sight (LOS) is often blocked, along with the fading environment and several other factors such as high mobility and uncoordinated medium access mechanism, which contributes significantly towards IEEE 802.11p standard performance degradation. Conversely, in LTE the base station nodes are located at higher positions which can help in leveraging the non-line of sight (NLOS) issues. Moreover, infrastructure-assisted scheduling and access control contributes significantly towards achieving the active road safety application requirements. However, it becomes increasingly difficult to meet the delay requirements in the presence of higher cellular network traffic load.

In the context of diverse and conflicting system performance requirements, it is important to understand the specific networking scenarios where one technology offers superior, inferior, or comparable performance over the other. Such studies are also important because of the fact that several standardization bodies and industrial/ academic consortiums like C2C-CC [36] and ETSI [37] openly endorse the feasibility of incorporating multiple radio access technologies-enabled OBUs in the near future. Furthermore, having a detailed insight into the capabilities and limitations of each technology would help in designing and developing novel network architecture and protocols where the disadvantages of one standard are offset by the advantages of the other. We present a detailed comparative study between IEEE 802.11p and LTE technology when applied to the vehicular networking environment. It would reveal an interesting insight into the impact of vehicle density and mobility on the IEEE 802.11p standard and the LTE technology and evaluates both standards in terms of delay, reliability, scalability, and mobility support. While many consider LTE technology as an alternative to the IEEE 802.11p standard, both standards actually complement each other in terms of application performance requirements.

\section{Performance evaluation}

This section describes the performance evaluation of IEEE 802.11p and LTE standards using the ns-3 (version 3.17) simulator [38]. Three performance metrics used to measure the performance are as follows:

(1). End-to-end delay, computed as the sum of all mean delays for each vehicle, normalized over the total number of flows in the network, where mean delay is defined as the ratio between the sums of all delays and the total number of received packets.

(2). Packet delivery ratio (PDR), computed as the ratio between the number of received packets and the transmitted packets during the simulation time.

(3). Throughput, defined as the sum of received data frame bytes at the destinations, averaged over the total number flows in the network.

In this paper, we primarily focus on investigating the performance of both technologies in the context of communication requirements posed by different types of vehicular networking applications. For this purpose the foremost emphasis is given on evaluating the delay, reliability, scalability, and mobility support criteria in realistic simulation environment with varying vehicle densities, transmission frequency, and average vehicle speed.

\subsection{Simulation environment}

The road network represents the urban scenario, a $5 \times 5$ Manhattan grid with 25 blocks, as illustrated in Figure 2. There are six vertical and six horizontal two-lane roads with $400 \mathrm{~m}$ spacing among them. The vehicles' routes and movement patterns are generated using the SUMO tool [39]. In order to study the impact of vehicle density, the numbers of vehicle are varied from 25 to 150 with an increment of 25. Similarly, to study the impact of speed, the average speed of the vehicle is varied from 20 to $100 \mathrm{~km} / \mathrm{h}$ with an increment of $20 \mathrm{~km} / \mathrm{h}$. Some may argue on the practicality of utilizing IEEE $802.11 \mathrm{p}$ in a dense road scenario (50 or above vehicles) and higher speeds. However, with a transmission range of $250 \mathrm{~m}$ and vehicle deployed over an area of $2 \mathrm{~km} \times 2 \mathrm{~km}$, it is quite real to have a few intersection or roundabout congested during the peak hours.

For IEEE $802.11 \mathrm{p}$-based vehicular network simulations, the communication range is set to $250 \mathrm{~m}$ (or 25 $\mathrm{dBm}$ ) with parameter values of EnergyDetectionThreshold and CCAModelThreshold set to -83 and $-86 \mathrm{dBm}$, respectively. For the propagation modeling, we combined three log distance propagation model and the Nakagami fading channel using $5.8 \mathrm{GHz}$ radio and data rate of 6 Mbps. By default, each vehicle transmits 256 B beacons at varying transmission frequency using an UDP-based 


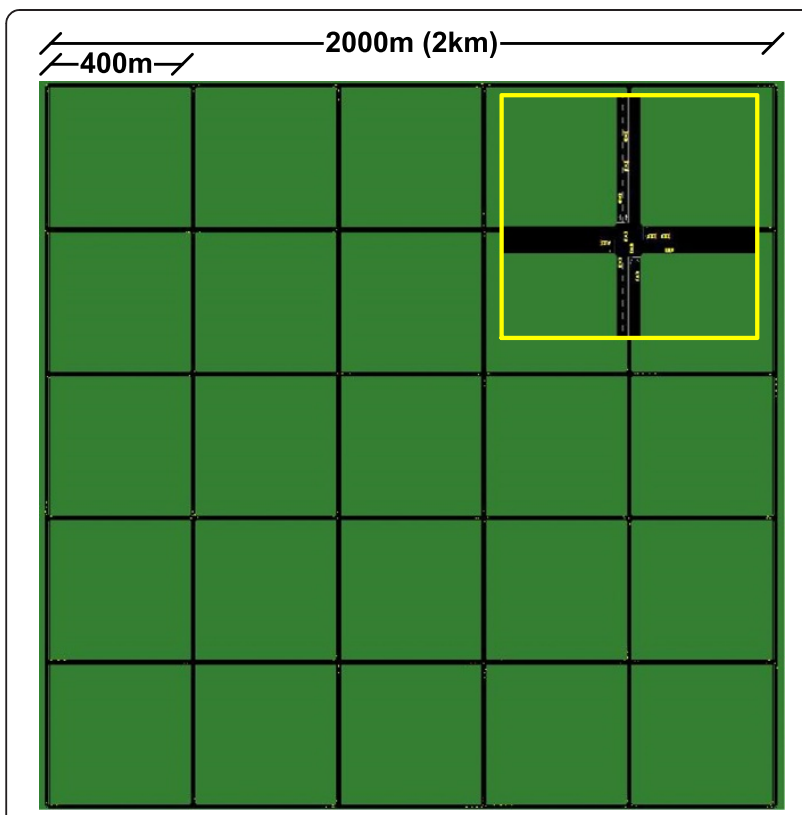

Figure 2 Road network, $5 \times 5$ Manhattan grid.

application. All vehicles communicate directly using singlehop broadcast without any assistance from the RSU.

As for the LTE technology simulations, we have utilized the LENA [40] module for ns-3. The RAN part of the LTE network constitutes a single eNB node (located at the lower left corner of the simulation area, coordinates $(0,0)$ ) representing the only LTE cell. The cell operates at the downlink (DL) frequency band of 2,110 MHz and uplink (UL) frequency band of 1,710 MHz, both with the bandwidth of $10 \mathrm{MHz}$ (or 50 resource blocks). The transmission power of the eNB and the vehicle (UE) nodes is set to 40 and $20 \mathrm{dBm}$, respectively.

The Friis path loss model is employed as the radio propagation model configured with an isotropic (omnidirectional) antenna. Finally, single-input, single-output (SISO) transmission mode and the Proportional Fair MAC scheduler are used in our simulations. In addition to the LTE radio access network, the simulation program also includes the EPC network with a single SGW/PGW node connected to a remote host $(\mathrm{RH})$ via a point-topoint link.

In our simulations there are mainly two types of vehicles, one that is configured to transmit beacons as per required by different vehicular networking applications in the domain of road safety, traffic efficiency, and infotainment, while few others transmit background traffic which emulates real-time video streaming application with the video bit rate of $440 \mathrm{kbps}$ and packet size of $1,203 \mathrm{~B}$. The arrival of background traffic is based on exponential distribution process with average arrival rate set to 1 , and the duration of initiated traffic lasts until the end of the simulation time. Both types of applications generate traffic from within the access network and a designated remote host in the Internet. The transmission method between the vehicles and the eNB node is single-hop unicast. Table 1 summarizes the simulation parameter settings for the IEEE 802.11p and LTE standards.

Table 1 Simulation parameters and values

\begin{tabular}{|c|c|c|}
\hline Simulation parameters & Values & \\
\hline Number of vehicles (periodic vehicular traffic) & $25,50,75,100,125,150$ & \\
\hline Vehicle average speed & $20,40,60,80,100 \mathrm{~km} / \mathrm{h}$ & \\
\hline Beacon transmission frequency & $1,4,8,10,20 \mathrm{~Hz}$ & \\
\hline Antenna type & Isotropic/omnidirectional & \\
\hline Simulation area & $2,000 \mathrm{~m} \times 2,000 \mathrm{~m}(2 \mathrm{~km} \times 2 \mathrm{~km})$ & \\
\hline Simulation duration & $100 \mathrm{~s}$ & \\
\hline Number of vehicles (background traffic) & 25 & \\
\hline Vehicular traffic (packet size) & $256 \mathrm{~B}$ & \\
\hline Background traffic (packet size) & 1,203 B & \\
\hline \multirow[t]{2}{*}{ Background traffic (data rate) } & $440 \mathrm{kbps}$ & \\
\hline & IEEE $802.11 p$ & LTE \\
\hline \multirow[t]{2}{*}{ Frequency } & $5.8 \mathrm{GHz}$ & $\mathrm{DL}-2,110 \mathrm{MHz}$ \\
\hline & & UL-1,710 MHz \\
\hline Channel bandwidth & $10 \mathrm{MHz}$ & $10 \mathrm{MHz}$ \\
\hline Transmission power & $25 \mathrm{dBm}$ & eNB $(40 \mathrm{dBm}) / \mathrm{UE}(20 \mathrm{dBm})$ \\
\hline Propagation loss model & Three log distance Nakagami fading & Friis \\
\hline Data rate/RB allocation & $6 \mathrm{Mbps}$ & $\mathrm{DL}(50) / \mathrm{UL}(50)$ \\
\hline
\end{tabular}

For all the other parameters, we used default values in ns-3 (3.17) network simulator. 


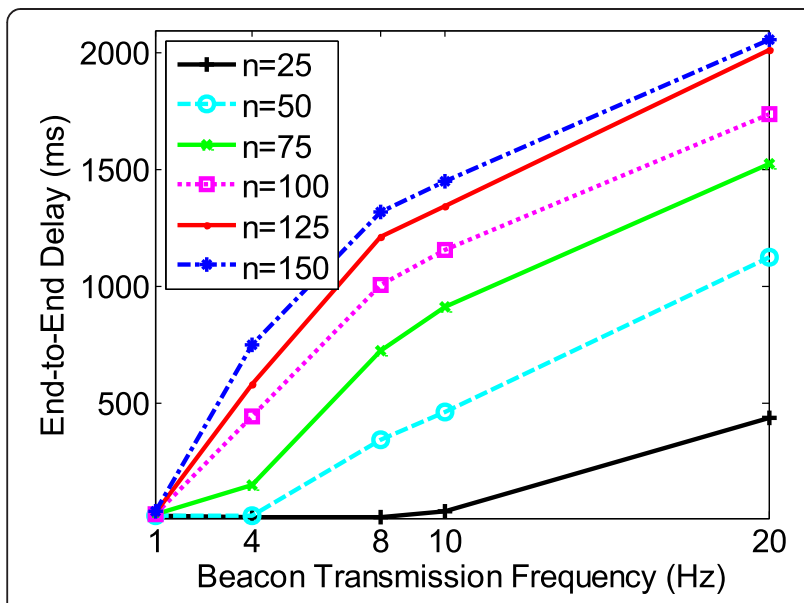

Figure 3 End-to-end delay (ms) vs. beacon transmission frequency $(\mathrm{Hz})$, IEEE $802.11 \mathrm{p}$-based vehicular network.

\subsection{Simulation studies}

Two performance evaluation studies are carried out. First is to study the impact of varying beacon transmission frequency (or number of beacons send per second) on the performance. Second is to study the impact of vehicle's average speed on the performance of both technologies. Even though in LTE-based vehicular network there are two types of vehicles representing vehicular traffic and background traffic, the focus of current study is on the performance evaluation of the vehicular traffic application only.

\subsubsection{Impact of varying beacon transmission frequency}

For the first set of simulation results, the beacon transmission frequency is varied. While the typical transmission frequencies are from 1 to $10 \mathrm{~Hz}$, few applications require this value to be $20 \mathrm{~Hz}$. The average speed is set to $20 \mathrm{~km} / \mathrm{h}$. Since most of the vehicular networking applications are time-critical, therefore, the end-to-end delay must be between 100 and $500 \mathrm{~ms}$. As shown in

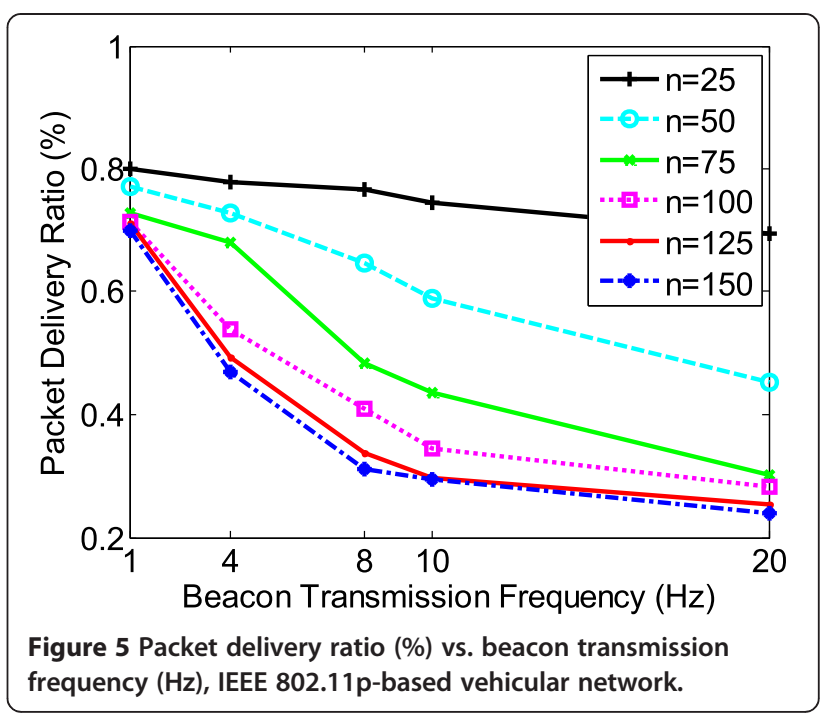

Figure 3 for the IEEE 802.11p network, the delay increases when the transmission frequency and number of vehicle increase. Sparse networks of sizes 25 and 50 exhibit better performance; however, as the network load increases, denser networks with higher transmission frequencies result in higher end-to-end delays.

Specifically, in Figure 4a,b we can notice that for 25vehicle network, more than $90 \%$ of the beacons had a delay of less than $100 \mathrm{~m}$. However, as the network size increases to 50 vehicles, on average only $40 \%$ of the beacons achieve a delay less than $100 \mathrm{~ms}$ for the typical transmission frequency of 1 to $10 \mathrm{~Hz}$.

Equally important is the reliability, which is often characterized by the PDR performance metric, illustrated in Figure 5. Even with the sparse network topologies and lower transmission frequencies, the successful packet delivery in the IEEE 802.11p network is considerably low due to severe congestion and packet losses.

Looking closely at Figure 6a,b which shows that for network size of 25 vehicles, on average only $50 \%$ of the
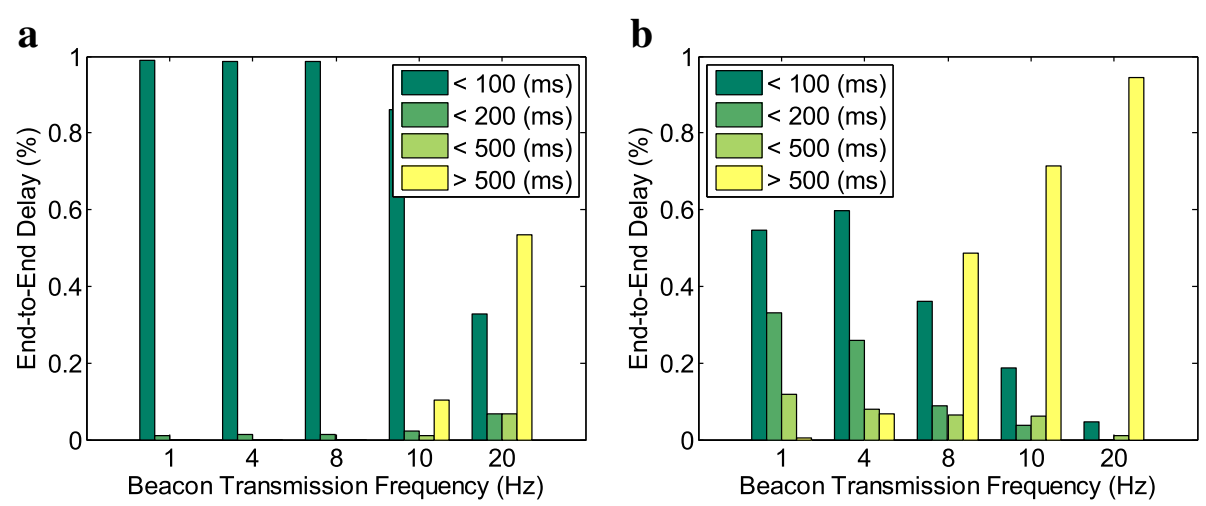

Figure 4 End-to-end delay (ms) distribution vs. beacon transmission frequency (Hz). IEEE 802.11 p-based vehicular network. (a) 25 vehicles. (b) 50 vehicles 

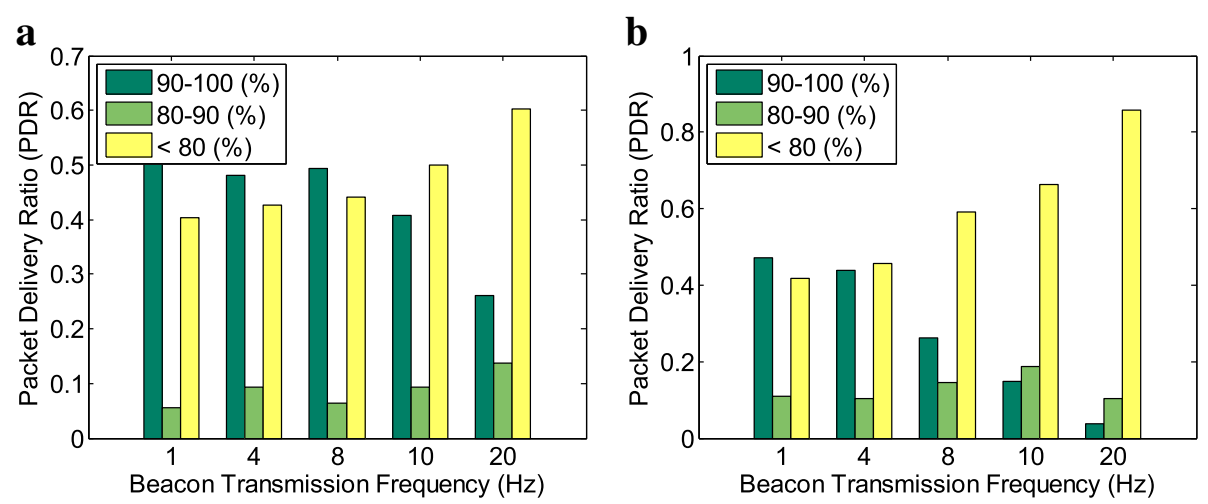

Figure 6 Packet delivery ratio distribution (\%) vs. beacon transmission frequency (Hz). IEEE 802.11 p-based vehicular network. (a) 25 vehicles. (b) 50 vehicles.

transmissions are successfully received for the most common transmission frequencies which drops further to $33 \%$ in the case of 50 vehicles. The increase in network size, along with larger number of packet transmissions per seconds, saturates the channel with traffic. Another contributing factor towards performance degradation is the lack of centralized coordination of the common channel among the contending vehicles. Consequently, the packet delivery ratio drops rapidly.

Figure 7 shows the aggregate throughput for the IEEE 802.11p network. Higher packet delivery ratio for the sparse network topologies contributes significantly towards attaining reasonable throughput. However, the throughput decreases significantly as the < network size, transmission frequency $>$ pair increases mainly due to higher number of packet loss.

Figure $8 \mathrm{a}$ shows the end-to-end delay for the LTE network with vehicular traffic application only. We observe that the increase in network size and transmission frequency increases the end-to-end delay. However, the

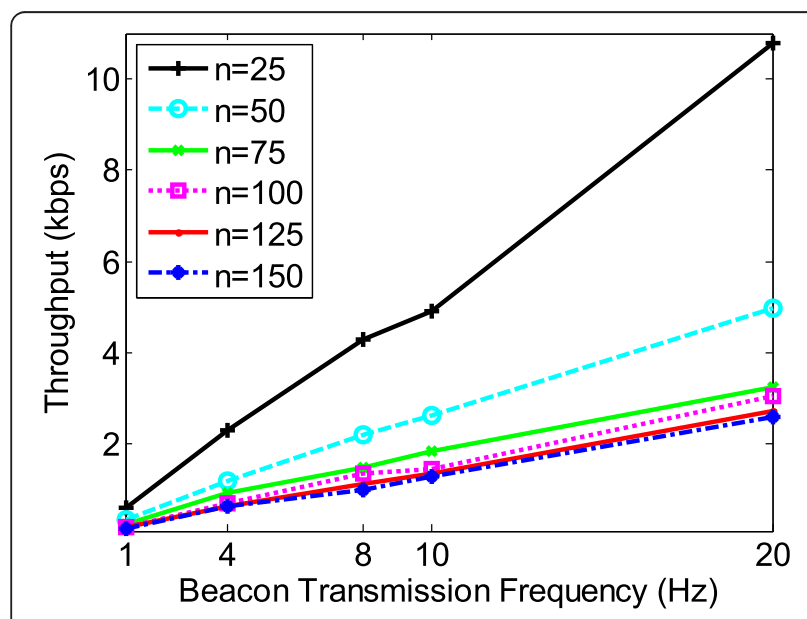

Figure 7 Throughput (kbps) vs. beacon transmission frequency $(\mathrm{Hz})$, IEEE 802.11p-based vehicular network.

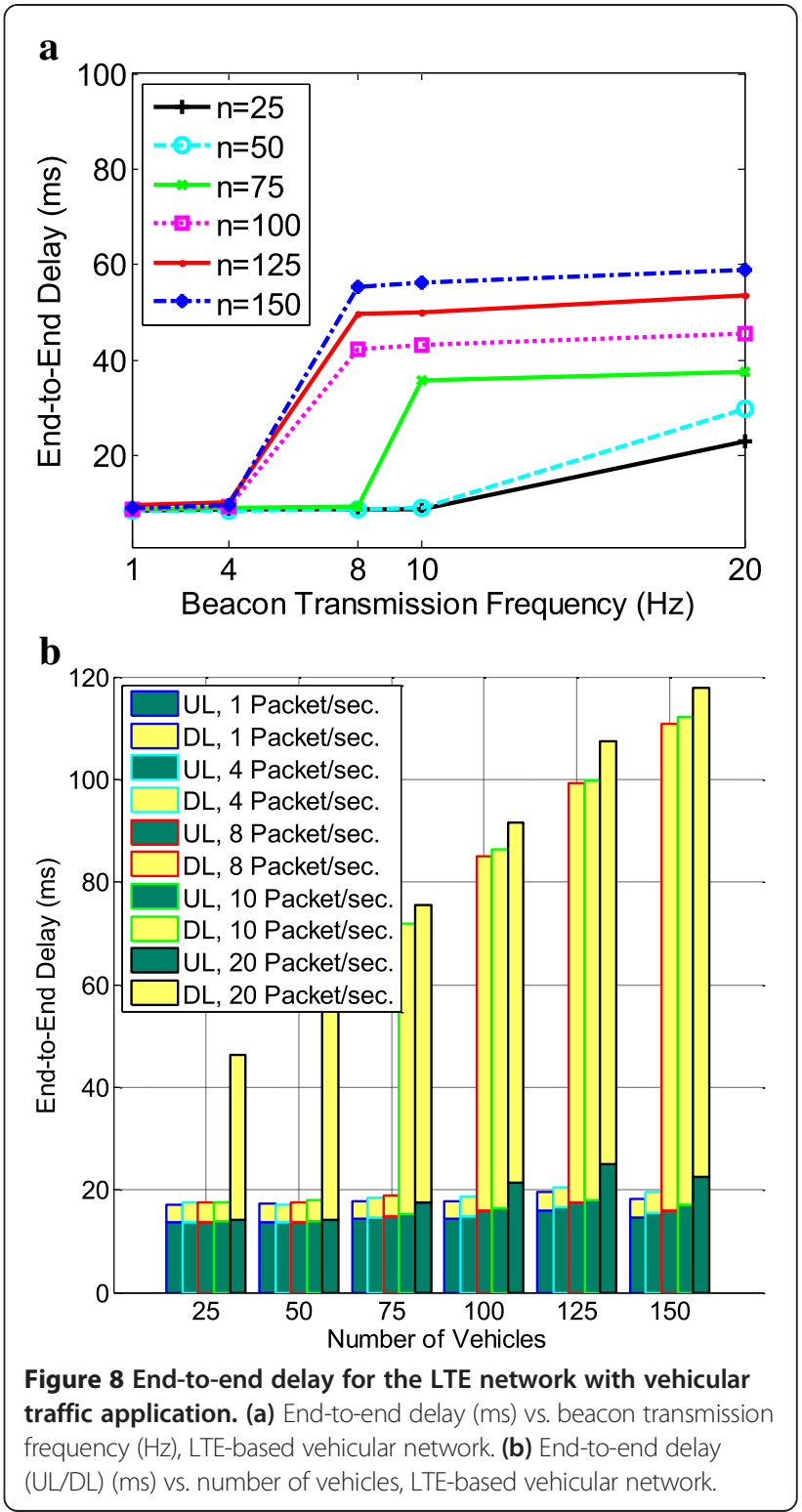


incurred average delay is significantly less and fulfills the delay requirements for all types of vehicular applications. Figure $8 \mathrm{~b}$ shows the uplink and downlink end-to-end delays experienced by the vehicles. It is interesting to know that for higher network load, the total delay however gradually increases. While the UL delays largely remain intact, the DL delays increase significantly as the number of vehicles and transmission frequency increases. The main factor contributing towards the DL delay is the choice of single-hop unicast transmission method employed to transmit beacons from eNB to all the individual UEs or vehicles. It further implies that even though for the given simulation scenario in which LTE meets the delay requirements, its performance is sensitive to the available network load.

The LTE network scenario outperforms IEEE 802.11p network in terms of PDR and throughput as shown in Figures 9 and 10, respectively. For sparse topologies, LTE network achieves $100 \%$ PDR. However, as the network size and transmission frequency pair increases, packet loss increases as well. Similarly, due to fewer packet losses, LTE network attains significant throughput, as indicated in Figure 10. The observation here is obvious, since more vehicles with higher transmission frequency and lower loss rate result in more packets delivered to the destination. The lower loss rate has negligible effect on the aggregate throughput because the beacon sizes are comparatively small in bytes. For the LTE networking scenario, much of the performance gain in terms of delay, PDR, and throughput is attributed towards infrastructure-assisted scheduling and centralized access control mechanism.

\subsubsection{Impact of varying average vehicle speed}

For the second set of simulation results, the average speed is varied and the beacon transmission frequency is set to $10 \mathrm{~Hz}$.

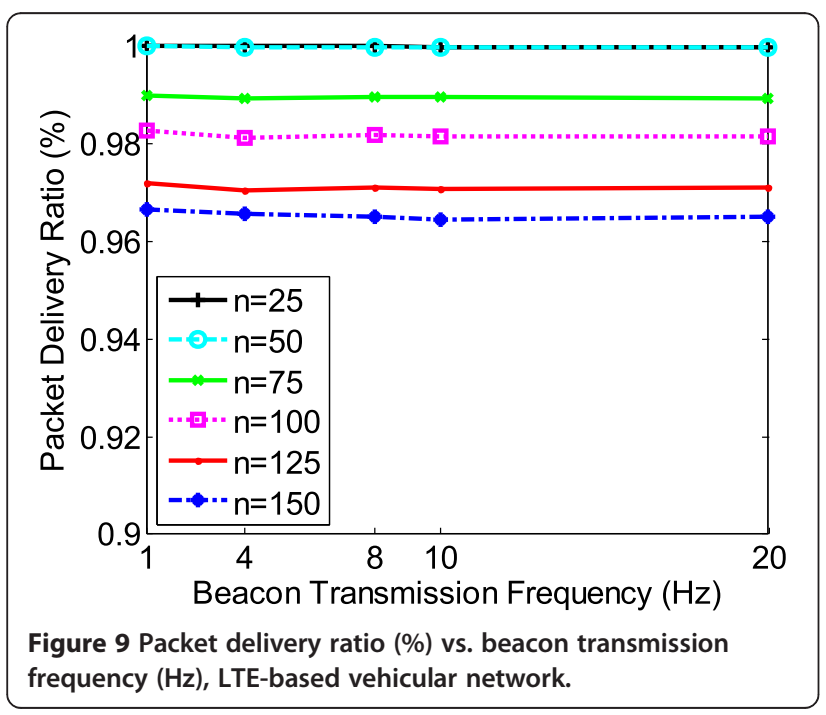

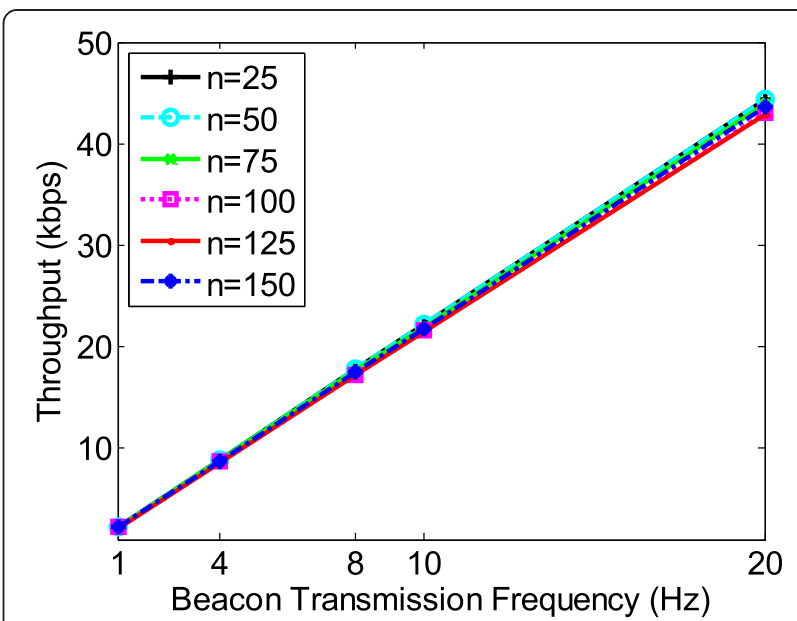

Figure 10 Throughput (kbps) vs. beacon transmission frequency $(\mathrm{Hz}), \mathrm{LTE}-$ based vehicular network.

Figure 11 shows the end-to-end delay for varying average vehicle speed values. Overall, as the vehicle speed increases, the delay increases. Vehicles spend more time in contending for the common channel as the result of larger neighborhood densities and because of the fact that they need to access the channel more frequently for higher transmission frequencies. The situation further exuberates due to the fact that different speed values tend to favor slower vehicles. This unfairness causes relatively faster vehicles to wait longer for accessing the channel. Once again, sparser topologies satisfy the delay requirements; for 25 -vehicle network, on average more than $75 \%$ of the beacons had a delay of less than $100 \mathrm{~ms}$. However, this is reduced to just $25 \%$ for 50 -vehicle network, as shown in Figure 12a,b.

Figure 13 illustrates the PDR as the average vehicle speed increases. The results indicate that for all the initial speeds value, there is a decline in successful reception of

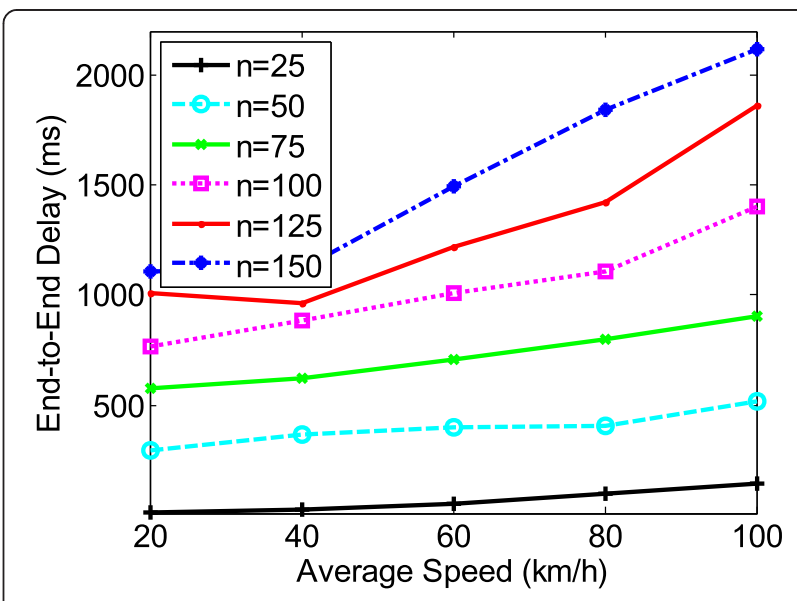

Figure 11 End-to-end delay (ms) vs. average speed (km/h), IEEE 802.11p-based vehicular network. 

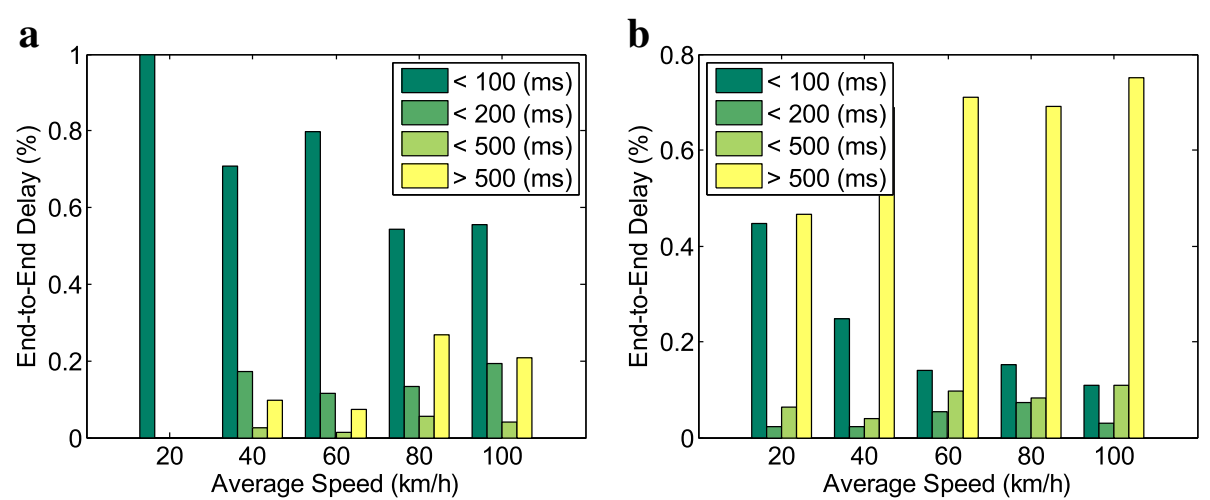

Figure 12 End-to-end delay (ms) distribution vs. average speed ( $\mathbf{k m} / \mathbf{h})$, IEEE $\mathbf{8 0 2 . 1 1 p - b a s e d ~ v e h i c u l a r ~ n e t w o r k . ~ ( a ) ~} 25$ vehicles. (b) 50 vehicles.

packets. There are two further observations. Firstly, the decline is more prominent in the case of sparser network, and secondly, as the average speed of the vehicle is increase further, vehicles tend to improve on the packet reception, which is more visible for networks with higher number of vehicles. These observations are attributed to following facts. Despite that slower-moving vehicles relatively gain more access to the channel, network fragmentation occurs more frequently within the sparse network. Conversely, this situation helps denser network to attain better reception ratio because network connectivity improves with larger number of vehicles. Figure 14a,b shows that even with sparser network and lower speed, the PDR is significantly low for the typical transmission frequency of $10 \mathrm{~Hz}$.

Figure 15 depicts the throughput as the average vehicle speed is varied, which essentially reflects on our previous observations. Denser networks tend to stay more connected as compared with the sparser networks. Therefore,

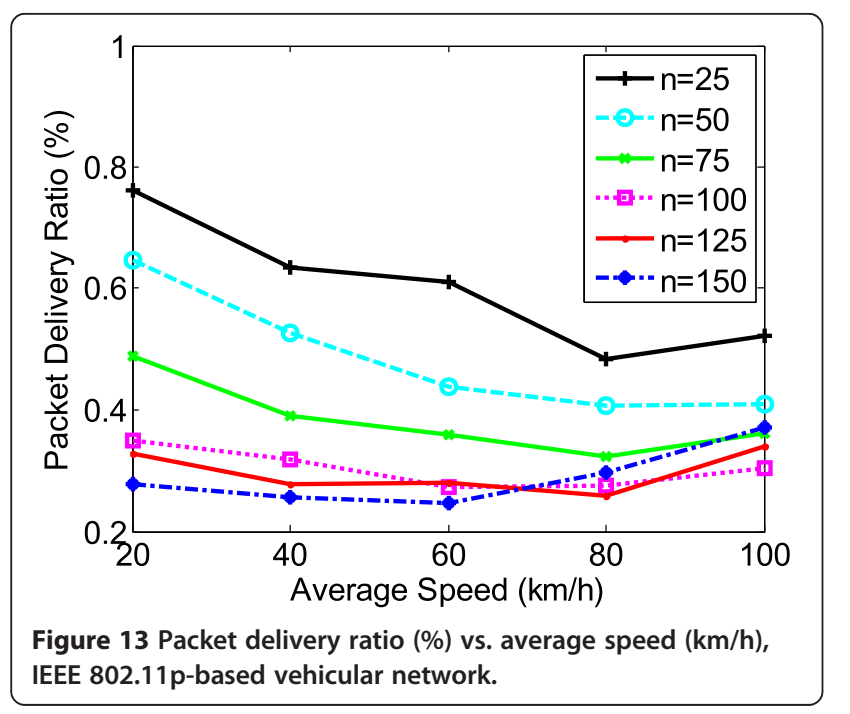

an improvement in successful packet reception results in better throughput.

Figures 16, 17 and 18 show the effect of average speed on the performance of LTE network in term of end-toend delay, PDR, and throughput, respectively. The performance for each metrics remained stagnant regardless of the vehicle speed for all the network sizes. As the network size increases, the delay increases while the PDR and throughput decrease slightly. The effect on throughput is less apparent mainly due to relatively smaller beacon sizes used by the vehicular traffic application. There are two possible reasons for the negligible effect of vehicle speed on the LTE performance. First is the use of simplified networking model with a single cell. Second is the lack of higher background traffic load, which would potentially saturate the LTE network. Using multicell configuration which takes into account intercell interferences and handover issues, larger network sizes with higher traffic load and inclusion of MBMS could give further insight on the LTE performance. However, this would significantly increase the simulation complexity and therefore deserved to be dealt independently and comprehensively in our future work.

\section{Discussion}

Our simulation study suggests that LTE standard scales better, delivers data reliably, and meets the latency requirements posed by several vehicular networking applications. The IEEE 802.11p standard, on the other hand, exhibits lower beacon latencies and higher delivery ratio/throughput in scenarios where there are fewer than 50 vehicles. However, as the number of vehicles increases, the standard is unable to support the performance requirements in different application categories.

There are mainly two reasons that necessitate a performance study of IEEE 802.11p and LTE standards. First, it provides a better insight in to the suitability of each standard for the given application and networking 

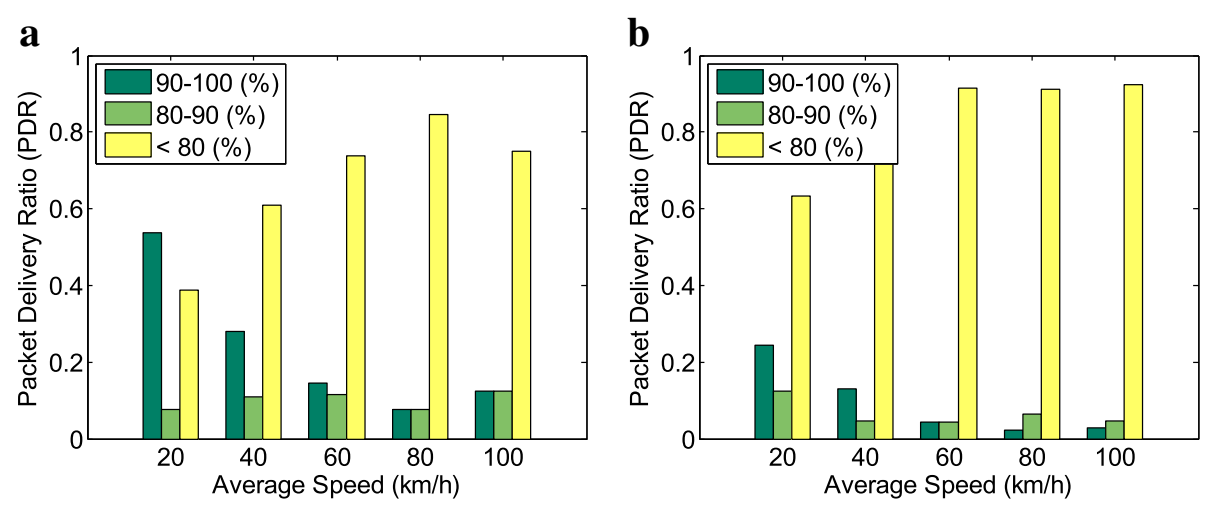

Figure 14 Packet delivery ratio (\%) distribution vs. average speed ( $\mathbf{k m} / \mathbf{h})$, IEEE $\mathbf{8 0 2 . 1 1 p - b a s e d ~ v e h i c u l a r ~ n e t w o r k . ~ ( a ) ~} 25$ vehicles. (b) 50 vehicles.

scenario. For example, it can be inferred that IEEE 802.11p is capable of handling active road safety and cooperative traffic efficiency latency and PDR requirements when vehicles are sparsely distributed within the same area. Similarly, despite that LTE meets most of the application requirements, its performance is sensitive to available network load and the number of cellular network users. This know-how is exceedingly important in the scenarios where vehicles are equipped with multiple radio access technologies (RAT) and has to intelligently select one over the other for data offloading. Second, more recently, several studies proposed heterogeneous or hybrid network architectures where the best from both infrastructure-based and infrastructure-less ad hoc networking domains are combined. Having a better understanding of both standards could help in designing and developing efficient network architecture and protocols for further performance gains.

The reason our study has practical relevance as well is due to the widespread proliferation of smartphones,

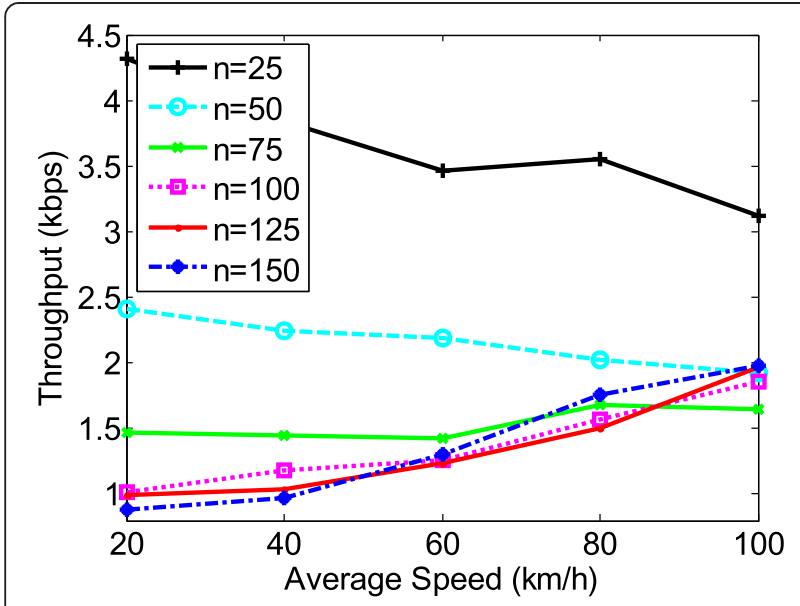

Figure 15 Throughput (kbps) vs. average speed (km/h), IEEE 802.11p-based vehicular network. tablets, and other handheld devices. These devices with variety of on-board sensors such as accelerometer, positioning capabilities via GPS, and a variety of connectivity modalities such Bluetooth, Wi-Fi Direct, and 3G/ 4G can provide a suitable platform for implementing real-time vehicular applications. The smartphones can share information like speed, location, and traffic status either via cellular network or device-to-device (D2D) communication.

Our findings that LTE can support most of the applications requirement in terms of delay and scalability not only validate the results given in [21] but also extend the previous study with metrics like reliability and mobility support under realistic simulation environment. Similarly, Vinel in [22,23] presents an analytical framework which compared IEEE 802.11p and LTE in the context of road safety applications. These studies made two conclusions. First, for an average contention window $(W=256)$ and network $(N=25)$ sizes, the probability that beacons are received within the delivery deadline $(100 \mathrm{~ms})$ varies

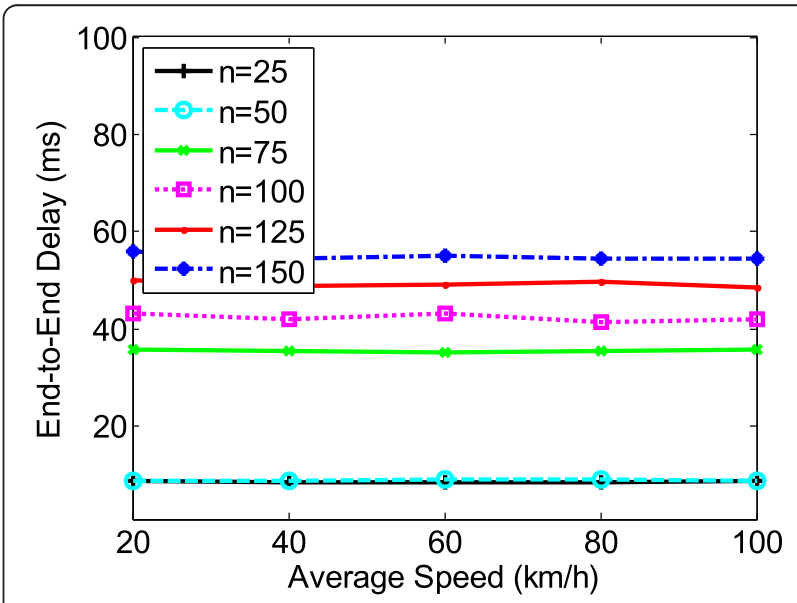

Figure 16 End-to-end delay (ms) vs. average speed (km/h), LTE-based vehicular network. 


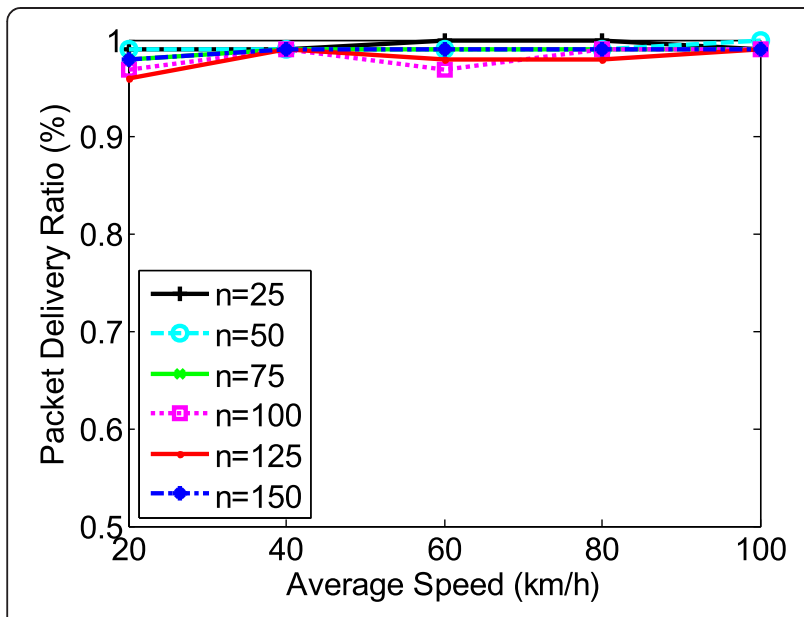

Figure 17 Packet delivery ratio (\%) vs. average speed $(\mathrm{km} / \mathrm{h})$, LTE-based vehicular network.

between $100 \%$ and $90 \%$. Moreover, this probability decreases to just $20 \%$ for $W=16$ and $N=50$. Second, the download unicast in LTE network is appropriate only for small number of vehicles (less than 100) in the cell. Our simulation study given in Figure 6a,b validates the former, while Figure 8a,b replicates the later conclusions which were obtained using theoretical analysis. Certainly, the differences in the specific simulation/analytical tools used to measure performance could contribute to the differences between the present study and that of Vinel [22,23]. For example, the conclusion made in $[22,23]$ that IEEE $802.11 p$ is a promising standard for active road safety applications is difficult to hold beyond network sizes of 50 vehicles, according to our studies.

The simulation environment along with parameter settings and assumptions exceedingly influences the performance evaluation study of IEEE 802.11p and LTE

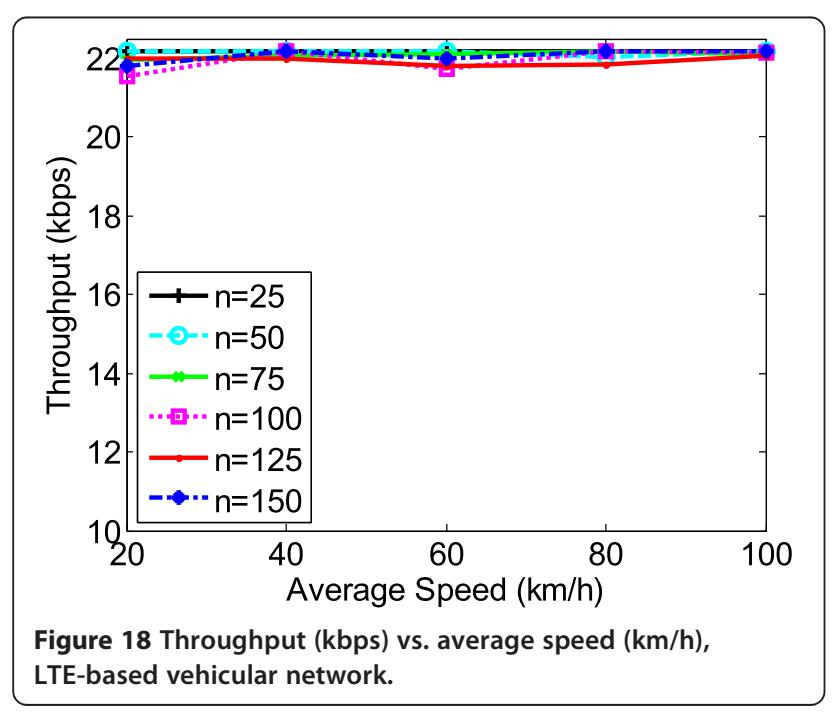

standards. There are number of ways the current study can be improved further. The foremost is the use of QoS-based scheduling algorithms; as suggested in the performance evaluation that for LTE networks, the latency increases as the network load increases. However, these issues can be resolved by properly differentiating and prioritizing background and vehicular network application traffic. Furthermore, in our study we considered the downlink unicast only; another avenue that needs further investigation is the use of downlink broadcast [22] via techniques like MBMS to improve downlink data channel capacity. Although the benefits are quite apparent, the associated overhead and communication cost has to be considered, evaluated, and reported. Lastly, in our simulation environment, the number of users with background traffic and the background traffic load remained unchanged. In order to better understand the effect of background traffic, it must be modeled by calculating spatial user and traffic distributions for LTE networks with time-varying usage intensities. Future research work would include performance evaluation with spatial and temporal traffic distributions which depend on the day of the week and the time of the day. One final avenue that remains to be explored is to evaluate performance based on real deployment of IEEE 802.11p and LTE-based vehicular networks.

\section{Conclusions}

In this paper we presented a comparative study between IEEE 802.11p and LTE for vehicular networking. Both standards are evaluated in terms of delay, reliability, scalability, and mobility support under various vehicular networking conditions and parameters settings. For the given simulation scenarios and network traffic load, our results show that LTE offered superior network capacity and mobility support as compared with the IEEE 802.11p standard. Thus, we can conclude that the LTE technology is suitable for most of the applications and use cases. While the attainable delay satisfies most of the vehicular network application requirements, we observed a tendency of increase in the delay as the network load increases (either increase in cellular traffic load or number of vehicles). As for IEEE 802.11p, the standard offered acceptable performance for sparse network topologies and typical transmission frequencies with limited mobility support. However, the performance is extremely sensitive to larger vehicle densities, traffic load, and vehicle speed. The performance gain in LTE network is attributed to fewer network elements and infrastructure-assisted scheduling and access control, whereas contributing factors towards performance degradation in IEEE 802.11p network include lack of coordinated channel access and distributed congestion control mechanisms based on in-network condition, such as traffic load and speed. Based on the above 
results, we further conclude that for smaller vehicle densities, IEEE 802.11p standard offers end-to-end delays less than $100 \mathrm{~ms}$ and throughput equivalent to $10 \mathrm{kbps}$ which satisfies the requirements posed by active road safety application and few of the more lenient cooperative traffic efficiency applications.

\section{Competing interests}

The authors declare that they have no competing interests.

\section{Acknowledgements}

This work was made possible by NPRP Grant No. 5-1080-1-186 from the Qatar National Research Fund (a member of The Qatar Foundation). The statements made herein are solely the responsibility of the authors.

\section{Received: 30 January 2014 Accepted: 8 May 2014}

Published: 30 May 2014

\section{References}

1. IEEE, IEEE Standard for Information Technology-Telecommunications and Information Exchange Between Systems-Local and Metropolitan Area Networks-Specific Requirements - Part II: Wireless LAN Medium Access Control (MAC) and Physical Layer (PHY) Specifications, Amendment 6: Wireless Access in Vehicular Environment (IEEE Computer Society, IEEE, NY, 2010)

2. 3GPP, Overview of 3GPP Release 8, 2008. http://www.3gpp.org/, Retrieved 2014-03-24

3. Y Park, S Kuk, H Kim, J Ha, C-JM Liang, K JeongGil, A feasibility study and development framework design for realizing smartphone-based vehicular networking systems. IEEE Transactions on Mobile Computing (TMC) 99, (2014). doi:10.1109/TMC.2014.2309959

4. CK Park, MW Ryu, KH Cho, Survey of MAC Protocols for Vehicular Ad Hoc Networks. Smart Computing Review, 2(4), 286-295 (2012). doi:10.6029/ smartcr.2012.04.005

5. KA Hafeez, L Zhao, Z Liao, BN Ma, Performance analysis of broadcast messages in VANETs safety applications, in Proceedings of the IEEE Global Telecommunications Conf. GLOBECOM 2010, Miami, FL, 6-10 December 2010

6. C Han, M Dianati, R Tafazolli, R Kernchen, Throughput analysis of the IEEE 802.11p enhanced distributed channel access function in vehicular environment, in Proceedings of the IEEE 72nd Vehicular Technology Conference Fall (VTC 2010-Fall), (Ottawa, ON). 6-9 September 2010

7. R Gallardo, D Makrakis, HT Mouftah, Performance analysis of the EDCA medium access mechanism over the control channel of an IEEE $802.11 \mathrm{p}$ WAVE vehicular network, in Proceedings of the IEEE International Conference on Communications (ICC 2009) (Dresden). 14-18 June 2009

8. J Misic, G Badawy, S Rashwand, VB Misic, Tradeoff issues for $\mathrm{CCH} / \mathrm{SCH}$ duty cycle for IEEE 802.11p single channel devices, in Proceedings of the IEEE Global Telecommunications Conference GLOBECOM 2010 (Miami, FL). 6-10 December 2010

9. JC Burguillo Rial, E Costa Montenegro, F Gil Castineira, P Rodriguez Hernandez, Performance analysis of IEEE 802.11p in urban environments using a multi-agent model, in Proceedings of the IEEE 19th International Symposium on Personal, Indoor and Mobile Radio Communications (PIMRC 2008), (Cannes). 15-18 September 2008

10. S Grafling, P Mahonen, J Riihijarvi, Performance evaluation of IEEE 1609 WAVE and IEEE 802.11p for vehicular communications, in Proceedings of the Second International Conference on Ubiquitous and Future Networks (ICUFN), (Jeju Island). 16-18 June 2010

11. S Eichler, Performance evaluation of the IEEE 802.11p WAVE communication standard, in Proceedings of the IEEE 66th Vehicular Technology Conference (VTC 2007-Fall), (Baltimore, MD). 30 September to 3 October 2007

12. IC Msadaa, P Cataldi, F Filali, Comparative study between $802.11 \mathrm{p}$ and mobile WiMAX-based V2I communication networks, in Proceedings of the Fourth International Conference on Next Generation Mobile Applications, Services and Technologies (NGMAST), (Amman). 27-29 July 2010

13. C Chou, C Li, W Chien, KA Lan, Feasibility study on vehicle-to-infrastructure communication: WiFi vs. WiMAX, in Proceedings of the Tenth International Conference on Mobile Data Management: Systems, Services and Middleware (MDM 2009), (Taipei). 18-20 May 2009
14. M Aguado, J Matias, E Jacob, M Berbineau, The WiMAX ASN network in the V2I scenario, in Proceedings of the 68th IEEE Vehicular Technology Conference (VTC 2008-Fall), (Calgary). 21-24 September 2008

15. D Carona, A Serrador, P Mar, R Abreu, N Ferreira, T Meireles, J Matos, J Lopes, A $802.11 \mathrm{p}$ prototype implementation, in Proceedings of the IEEE Intelligent Vehicles Symposium (IV), (San Diego, CA). 21-24 June 2010

16. A Paier, R Tresch, A Alonso, D Smely, P Meckel, Y Zhou, N Czink, Average downstream performance of measured IEEE 802.11p infrastructure-tovehicle links, in Proceedings of the IEEE International Conference on Communications Workshops (ICC), (Capetown). 23-27 May 2010

17. F Bai, H Krishnan, Reliability analysis of DSRC wireless communication for vehicle safety applications, in Proceedings of the IEEE Intelligent Transportation Systems Conference (ITSC 2006), (Toronto). 17-20 September 2006

18. G Araniti, C Campolo, M Condoluci, A lera, A Molinaro, LTE for vehicular networking: a survey. IEEE Commun. Mag. 51(5), 148-157 (2013)

19. HY Kim, DM Kang, JH Lee, TM Chung, A performance evaluation of cellular network suitability for VANET. World Academy of Science, Engineering and Technology, International Science Index 64, 6(4), 1023-1026 (2012)

20. S Kato, M Hiltunen, K Joshi, R Schlichting, Enabling vehicular safety applications over LTE networks, in Proceedings of the International Conference on Connected Vehicles (ICCVE 2013), (Las Vegas, NV). 2-6 December 2013

21. K Trichias, Modeling and evaluation of LTE in intelligent transportation systems, (University of Twente and TNO, 2011), Enschede, Netherlands. Master of Science (M.Sc.) thesis

22. A Vinel, 3GPP LTE versus IEEE 802.11p/WAVE: which technology is able to support cooperative vehicular safety applications? IEEE Wireless Commun. Lett. 1(2), 125-128 (2012)

23. A Vinel, B Bellalta, N Chilamkurti, Y Koucheryavy, Scalability analysis of infrastructure networks for vehicular safety applications, in Proceedings of the International Conference on Connected Vehicles (ICCVE 2012), (Beijing). 12-16 December 2012

24. M Phan, R Rembarz, S Sories, A capacity analysis for the transmission of event and cooperative awareness messages in LTE networks, in Proceedings of the 18th ITS WORLD CONGRESS, (Orlando, FL). 16-20 October 2011

25. PC Gil, CC Gil, JM Gil, Design and implementation of an application for deploying vehicular networks with smartphones. Hindawi Int J Distributed Sensor Networks (IJDSNs) (2013). http://dx.doi.org/10.1155/2013/834596

26. M Ambrosin, A Bujari, M Conti, F De Gaspari, CE Palazzi, Smartphone and laptop frameworks for vehicular networking experimentation, in Proceedings of the 2013 IFIP Wireless Days (WD), (Valencia). 13-15 November 2013

27. SM Tornell, CT Calafate, J-C Cano, P Manzoni, M Fogue, FJ Martinez, Evaluating the feasibility of using smartphones for ITS safety applications, in Proceedings of the 2013 IEEE 77th Vehicular Technology Conference (VTC 2013Spring), (Dresden). 2-5 June 2013

28. H Abid, TC Chung, S Lee, S Qaisar, Performance analysis of LTE smartphones-based vehicle-to-infrastructure communication, in Proceedings of the 9th International Conference on Ubiquitous Intelligence Computing and Autonomic Trusted Computing (UIC/ATC), (Fukuoka). 4-7 September 2012

29. A Corti, V Manzoni, SM Savaresi, M Santucci, O Di Tanna, A centralized real-time driver assistance system road safety based on smartphone, in Proceedings of the First Workshop on Pervasive Urban Applications (San Francisco, CA). 12-15 June 2011

30. Q Zhao, Y Zhu, C Chen, H Zhu, L Bo, When 3G meets VANET: 3G-assisted data delivery in VANETs. IEEE SENSORS J. 13, 10 (2013)

31. S Busanelli, F Rebecchi, M Picone, N lotti, G Ferrari, Cross-network information dissemination in vehicular ad hoc networks (VANETs): experimental results from a smartphone-based testbed. Future Internet 5(3), 398-428 (2013). doi:10.3390/fi5030398

32. G Remy, SM Senouci, F Jan, Y Gourhant, LTE4V2X: LTE for a centralized VANET organization, in Proceedings of the IEEE Global Telecommunications Conf. (GLOBECOM 2011), (Houston, TX). 5-9 December 2011

33. R Sivaraj, AK Gopalakrishna, MG Chandra, P Balamuralidhar, QoS-enabled group communication in integrated VANET-LTE heterogeneous wireless networks, in Proceedings of the IEEE 7th International Conference on Wireless and Mobile Computing, Networking and Communications (WiMob), (Wuhan). 10-12 October 2011

34. K Katsaros, M Dianati, L Le, Effective implementation of location services for VANETs in hybrid network infrastructures, in Proceedings of the IEEE ICC 2013 
Workshop on Emerging Vehicular Networks: V2VN2l and Railroad Communications, (Budapest). 9-13 June 2013

35. G Karagiannis, O Altintas, E Ekici, G Heijenk, B Jarupan, K Lin, T Weil, Vehicular networking: a survey and tutorial on requirements. Architectures, challenges, standards. IEEE Commun. Surveys Tutorials 13(4), 584-616 (2011)

36. R Baldessari, B Bödekker, A Brakemeier, M Deegener, A Festag, W Franz, A Hiller, C Kellum, T Kosch, A Kovacs, M Lenardi, A Lübke, C Menig, T Peichl, M Roeckl, D Seeberger, M Strassberger, H Stratil, HJ Vögel, B Weyl, W Zhang, Car to Car Communication Consortium, Car to Car Communication Consortium manifesto: overview of the C2C-CC system, C2C-CC, Version 1.1. June 2009

37. ETSI, ETSI TR 102 638, Intelligent Transport System (ITS); vehicular communications; basic set of applications; definition, ETSI specification TR 102 638, Version 1.1.1. Technical Report. June 2009

38. ns-3. ns-3 network simulator. http://www.nsnam.org/, ns-3.17 release. Accessed 18 August 2013

39. D Krajzewicz, J Erdmann, M Behrisch, L Bieker, Recent development and applications of SUMO - simulation of urban mobility. Int. J. Advances Syst. Measure 5(3\&4), 128-138 (2012)

40. M Mezzavilla, M Miozzo, M Rossi, N Baldo, M Zorzi, A lightweight and accurate link abstraction model for system-level simulation of LTE networks in ns-3, in Proceedings of the ACM International Conference on Modeling, Analysis and Simulation of Wireless and Mobile Systems (MSWiM), (Paphos, Cyprus Island). 21-25 October 2012

doi:10.1186/1687-1499-2014-89

Cite this article as: Hameed Mir and Filali: LTE and IEEE $802.11 \mathrm{p}$ for

vehicular networking: a performance evaluation. EURASIP Journal on Wireless Communications and Networking 2014 2014:89.

\section{Submit your manuscript to a SpringerOpen ${ }^{\circ}$ journal and benefit from:}

- Convenient online submission

- Rigorous peer review

- Immediate publication on acceptance

- Open access: articles freely available online

- High visibility within the field

- Retaining the copyright to your article

Submit your next manuscript at $\gg$ springeropen.com 